\title{
DEPOSICIÓN DE CAPAS FUNCIONALES SOBRE ESMALTES CERÁMICOS MEDIANTE LA TÉCNICA SOL-GEL (REVISIÓN)
}

\section{FunCTIONALIZED ENAMEL OF CERAMIC TILES BY SOL GEL TECHNIQUE (REVIEW)}

\author{
Santiago Betancourt-Parra ${ }^{1, *}$, Manuel A. Domínguez-Ortiz ${ }^{1}$, Diana M. \\ Mosquera-Palacio $^{1}$, Juliana Herrera-Guerra ${ }^{1}$, Carlos M. Ríos-Rendón ${ }^{2}$, \\ Carlos E. Villa ${ }^{3}$
}

\section{Resumen}

La funcionalización de esmaltes para la industria de las baldosas cerámicas ha sido un frente de investigación importante en los últimos 15 años. Diferentes investigadores han centrado sus esfuerzos en conseguir superficies con atributos funcionales que incrementen el valor agregado del producto y a su vez aporten respuestas a las necesidades tecnológicas de nuestros tiempos. El presente artículo se centra en hacer una revisión de la literatura científica dedicada a la obtención de superficies funcionales por medio de la técnica de sol-gel, la cual es apta para la fabricación de soluciones que se depositan formando capas finas sobre los esmaltes de cerámica tradicional con el fin de aportar características funcionales al mismo. El documento presenta las principales soluciones alcóxidicas usualmente empleadas, las diferentes técnicas de deposición haciendo énfasis en los hallazgos experimentales obtenidos por los diferentes autores, y presenta una síntesis de los efectos funcionales hasta la fecha obtenidos por medio de la técnica.

Palabras clave: esmaltes cerámicos, esmaltes funcionales, sol-gel, cerámica.

\section{Abstract}

Functionalized enamels for the ceramic tile industry has been a research topic since the past 15 years. Different researchers have focused their efforts on achieving surfaces with functional attributes that increase product value and provide technical solutions for the technological needs of our times. This article presents a review of the scientific literature dedicated to obtaining functional surfaces by means of a sol gel technique, that provides a means for deposition and formation of thin layers on traditional ceramic enamels in order to provide functional characteristics. The document presents: typical used alcoxidic solutions, the different deposition techniques emphasizing the experimental findings obtained by the authors, and presents a synthesis of the functional effects obtained by means of the technique.

Keywords: Ceramic enamel, Functional enamel, sol gel, ceramic.

\footnotetext{
$\overline{1, *}$ Grupo de Investigaciones sobre Nuevos Materiales (GINUMA), Universidad Pontificia Bolivariana (UPB), Colombia. Autor para correspondencia santiago.betancourt@upb.edu.co (D) http://orcid.org/0000-0003-4474-2447,

(D) http://orcid.org/0000-0003-2167-7400, (D) http://orcid.org/0000-0003-0401-3557,

(D) http://orcid.org/0000-0002-5636-7210

${ }^{2}$ Dirección de desarrollo de producto, Eurocerámica, Colombia, (D) http://orcid.org/0000-0001-6816-4296

${ }^{3}$ Dirección técnica de planta, SENCO, Colombia, (D) http://orcid.org/0000-0002-4971-689X
}

Recibido: 16-11-2018, aprobado tras revisión: 13-12-2018

Forma sugerida de citación: Betancourt-Parra, S.; Domínguez-Ortiz, M. A.; Mosquera-Palacio, D. M.; Herrera-Guerra, J.; Ríos-Rendón, C. M. y Villa, C. E. (2019). «Deposición de capas funcionales sobre esmaltes cerámicos mediante la técnica sol-gel (revisión)». IngEnius. N. ${ }^{\circ}$ 21, (enero-junio). pp. 9-20. DoI: https://doi.org/10.17163/ings.n21.2019.01. 


\section{Introducción}

El esmalte cerámico es la capa de naturaleza vítrea que se deposita sobre la superficie de diferentes sustratos, generalmente también de naturaleza cerámica, para proporcionar atributos estéticos y proteger la superficie gracias a sus propiedades físicas. Un campo amplio de aplicación de los esmaltes es en las baldosas cerámicas, usadas para pavimentos y revestimientos en el sector de la construcción [1,2]. En tal caso, en las baldosas se pueden reconocer por tres capas claramente definidas, ver Figura 1, que son: i) la pasta, que es la encargada de servir de soporte, es la que tiene el mayor espesor y cuerpo en la baldosa, ii) el engobe que es un revoque de arcilla que posibilita la unión entre el esmalte y la pasta, aproximadamente 100 a $200 \mu \mathrm{m}$, iii) el esmalte, el cual es una capa de muy baja porosidad, de aproximadamente 75 a $300 \mu \mathrm{m}$ con las características mencionadas [3].

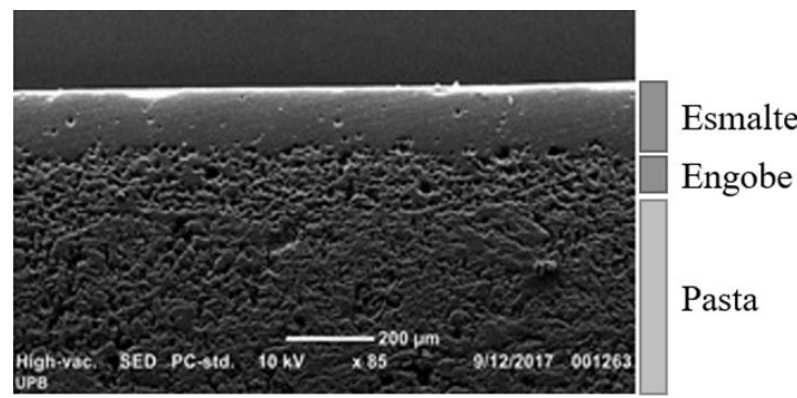

Figura 1. Corte en sección transversal de una baldosa cerámica.

Además de aportar características estéticas decorativas, los esmaltes de dichas baldosas pueden ser usados para otras posibilidades funcionales. Entre las funcionalidades que se les desea mejorar a las superficies se encuentran características tales como: autolimpiantes, almacenadoras de energía, fotocatalíticas, antibacterianos, fungicidas, alta resistencia al rayado, inhibidoras de la formación de hielo, que absorben o emiten sustancias, superficies luminiscentes y hasta recubrimientos que puedan cambiar propiedades bajo el efecto de un estímulo [4]. No obstante, muchos de los parámetros necesarios para conseguir una aplicación a escala industrial no son lo suficientemente estables, y aún se encuentran en investigación [5]. Siendo para la industria cerámica todo un reto, dado que la incorporación de nuevas tecnologías, trae consigo adecuaciones en los ciclos de producción y las condiciones rutinarias de fabricación que se traducen en altas inversiones económicas.

A nuestro entendimiento, existen varias técnicas para proporcionar los atributos funcionales a los esmaltes entre las que se encuentran: i) la modificación de la composición del esmalte mediante la incorporación de sustancias en la mezcla del esmalte antes de quema, ii) la modificación física de la superficie usando plasma o medios de deposición química en fase vapor, iii) la deposición química de soluciones líquidas, siendo la técnica de sol-gel una de las más representativas de esta familia.

La técnica de síntesis de materiales por medio de sol-gel ha sido ampliamente descrita como una técnica probada para la obtención de materiales vítreos y cerámicos [6-10]. Mediante reacciones de hidrólisis y condensación que ocurren en procesos químicos húmedos, se consiguen transformaciones moleculares de las sustancias precursoras en redes de óxidos [11].

La siguiente revisión se centra específicamente en compilar información científica relacionada con la deposición de soluciones químicas por medio de la técnica de sol-gel en esmaltes vítreos de baldosas cerámicas con el fin de incrementar propiedades o aportar atributos funcionales a la superficie esmaltada. En la Figura 2 se realiza un reporte gráfico de la evolución de publicaciones por año realizadas sobre el tema en bases de datos científicas online y libros especializados citados en la literatura consultada, entre las que se encuentran Google Scholar, Science Direct, Wiley Online Library, Springer Link, Repositorio Institucional UN, American Institute of Physics, Taylor \& Francis Online, ACS Publications.

La revisión se realizó para el período comprendido entre el año 2000 y 2017. Siendo el 2009-2017 el de mayor número de publicaciones acerca del tema. En este reporte, primero, se concentra en presentar los diferentes tipos de soluciones alcóxidicas usualmente empleadas. Luego, se enfoca en una descripción de las diferentes técnicas de deposición haciendo énfasis en los hallazgos experimentales obtenidos por los diferentes autores. Finaliza, presentando una síntesis de los efectos funcionales.

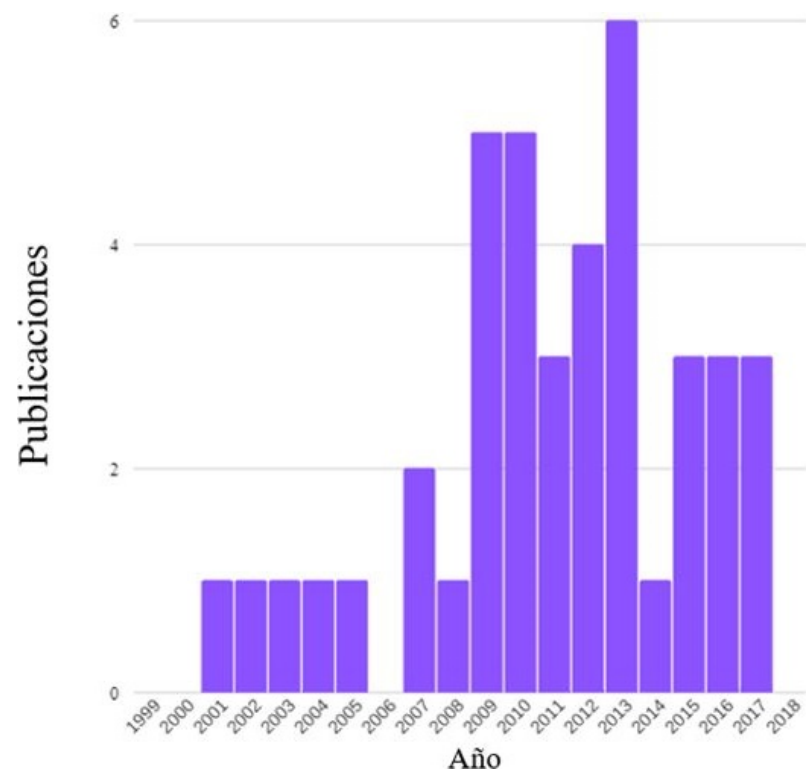

Figura 2. Relación de publicaciones por año. 


\section{Precursores}

En la Tabla 1 se presentan las soluciones químicas precursoras comúnmente empleadas para hacer recubrimientos sobre esmaltes mediante la técnica de sol-gel y sus correspondientes estructuras. Los precursores (reactivos de partida) son un alcóxido de fórmula química típica R-O-M, los cuales son compuestos que presentan un ion metálico (u otro catión) representado por la letra $\mathrm{M}$, una molécula de oxígeno representada por la letra $\mathrm{O}$ y un grupo alquilo $\mathrm{R}$. Las reacciones generales se expresan en la Tabla 2 acorde con lo indicado en [12].

Tabla 1. Soluciones químicas precursoras

$\begin{array}{lll}\text { Nombre } & \text { Formula molecular } \\ \text { Propóxido } \\ \text { de circonio } \\ \text { Etóxido de }\end{array}$

Tabla 2. Reacciones generales proceso sol-gel

\begin{tabular}{c}
\hline Hidrólisis \\
\hline $\mathrm{M}-(\mathrm{OR})_{\mathrm{x}}+\mathrm{H}-\mathrm{O}-\mathrm{H} \rightarrow \mathrm{HO}-\mathrm{M}(\mathrm{OR})_{\mathrm{x}-1}+\mathrm{H}-\mathrm{OR}$ \\
\hline Condensación \\
\hline Alcoxolación $_{(\mathrm{OR})_{\mathrm{x}-1}-\mathrm{M}-\mathrm{OH}+\mathrm{RO}-\mathrm{Me}-(\mathrm{OR})_{\mathrm{x}-1} \rightarrow(\mathrm{RO})_{\mathrm{x}-1}}$ \\
$-\mathrm{Me}-\mathrm{O}-\mathrm{M}-(\mathrm{OR})_{\mathrm{x}-1}+\mathrm{ROH}$ \\
Oxalación $_{(\mathrm{RO})_{\mathrm{x}-1}-\mathrm{M}-\mathrm{OH}+\mathrm{HO}-\mathrm{M}-(\mathrm{OR})_{\mathrm{x}-1} \rightarrow(\mathrm{RO})_{\mathrm{x}-1}}-\mathrm{M}-\mathrm{O}-\mathrm{M}-(\mathrm{OR})_{\mathrm{x}-1}+\mathrm{HOH}$ \\
Convenciones: \\
$\mathrm{M}: \mathrm{Si}, \mathrm{Ti}, \mathrm{Zr}_{\mathrm{H}} \mathrm{Hf}, \mathrm{Ta}, \mathrm{Nb}, \mathrm{Al}, \mathrm{Zn}$, etc. \\
$\mathrm{R}: \mathrm{CH}_{3}, \mathrm{C}_{2} \mathrm{H}_{5}, \mathrm{C}_{3} \mathrm{H}: 7$, etc.
\end{tabular}

Una vez reaccionan, los precursores forman coloides, los cuales son una suspensión, en la que la fase dispersada es pequeña (1-1000 nm), las fuerzas gravitacionales son despreciables y las interacciones son dominadas por fuerzas de corto rango (las fuerzas de atracción de Van der Waals y las cargas superficiales). El sol es una suspensión coloidal de partículas sólidas dentro de un líquido.

El punto de gel es el momento en cual las partículas enlazadas de óxido se extienden por el recipiente que contiene el coloide. Durante la gelación, la viscosidad se incrementa hasta alcanzar a formar un sólido que adapta la forma del molde. En este punto, el líquido del poro está constituido principalmente de alcohol y agua, y el coloide se convierte en alcogel, el cual puede ser removido del recipiente y puede sostenerse por sí mismo [13].

En la Figura 3, se muestra una representación esquemática elaborada por [6]. Los aerogeles por su lado se obtienen cuando el poro que está lleno de líquido es reemplazado por aire sin alterar la estructura o el volumen del cuerpo del gel.

Las reacciones en el proceso sol-gel dependen de varios parámetros, por ejemplo:

- Composición y concentración de los precursores (alcóxido y solvente)

- Cantidad de agua adicionada

- Tipo y concentración del catalizador usado

- Aditivos adicionales como desecantes

- Secuencia en la cual los componentes son adicionados

- Tiempo de mezcla

- Tiempo de añejamiento

- Condiciones de mezclado (eficiencia de la mezcla, agitación por ultrasonido, atmósfera)

- Temperatura. 


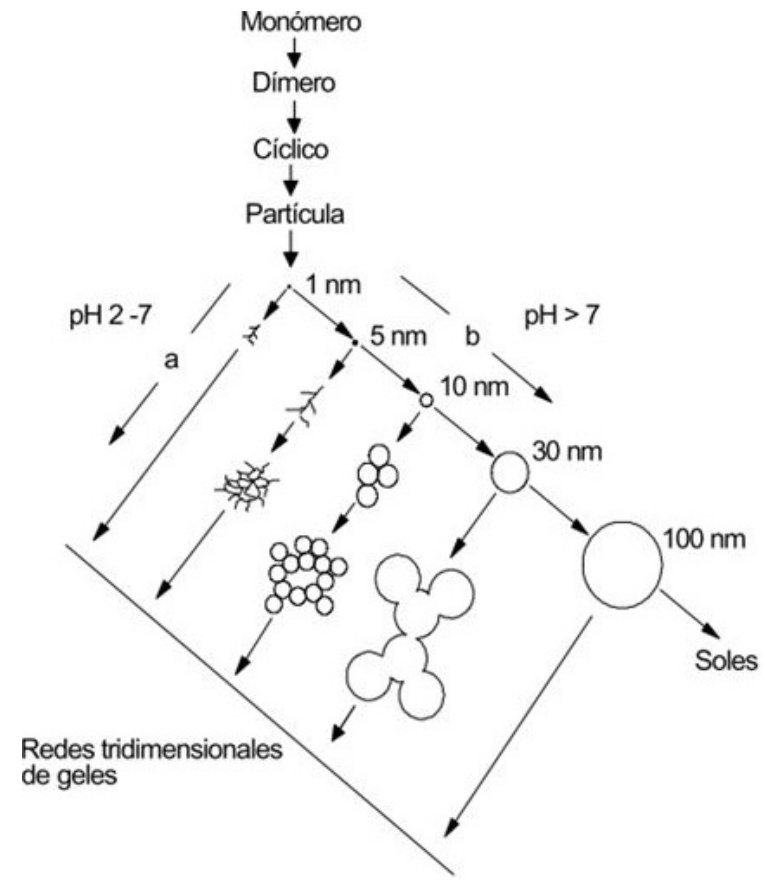

Figura 3. Representación esquemática del proceso sol-gel, tomado de [6].

\section{Técnicas de deposición}

En relación con las técnicas de deposición, a continuación, se presentan los hallazgos resaltados en la información estudiada, que se complementan con la información presentada en la Tabla 3 en la que se resumen características de las técnicas reportadas.

\subsection{Rotacional (Spin)}

Esta técnica basa su funcionamiento en la deposición de pequeñas cantidades de solución en la superficie de un sustrato, mientras esta gira a alta velocidad, de forma tal que la solución se dispersa por acción de la fuerza centrífuga, formando una película líquida homogénea. La solución evapora rápidamente el solvente y las moléculas del óxido se depositan sobre el sustrato. El espesor de la película es una función que depende directamente de las variables: velocidad de rotación, la viscosidad, el contenido de solvente y sus propiedades al evaporarse.

Existen varios modelos matemáticos para la variación del espesor descritos ampliamente en [12-17]. Con esta técnica se pueden obtener recubrimientos de capa delgada con espesor entre 0,1 y $10 \mu \mathrm{m}$ [18].

Es ampliamente usada en aplicaciones donde se requiera la formación de películas uniformes, es decir, con espesores homogéneos y de baja rugosidad [19]. Por esta razón, se emplea en la fabricación de microcomponentes electrónicos y ha resultado de interés para la obtención de altos brillos en los esmaltes de las baldosas dado que al reducir la rugosidad de la superficie aumenta el brillo de la misma [2].

\subsection{Inmersión (Dip)}

La técnica de deposición por inmersión (o en inglés Dip coating) basa su procedimiento en la inmersión total del sustrato en la solución, para luego ser removido a una velocidad controlada [20,21], bajo condiciones ambientales. Acorde con algunos investigadores es considerada como la técnica más fácil para depositar películas en la superficie de un sustrato, los cuales pueden ser tanto planos como curvos [11]. Incluso afirman que puede ser implementada para superficies con áreas en ordenes de metros cuadrados.

El espesor de la película depende directamente de la velocidad de retracción del sustrato y la viscosidad de la solución, siendo bajas velocidades las apropiadas para obtener películas delgadas de espesor aproximado entre 0,3 y $3 \mu \mathrm{m}$, y rugosidad entre 60 y $690 \mathrm{~nm}$ [18].

En el año 2007, Kuisma et al. [22], estudiaron la capacidad de limpieza de películas delgadas de $\mathrm{ZrO}_{2}$ y $\mathrm{TiO}_{2}$ depositadas por inmersión sobre superficies de baldosas cerámicas esmaltadas de $3,0 \times 3,0 \mathrm{~cm}$; investigación que mostró por medio de mediciones topográficas, que cuanto más áspero era el esmalte cerámico, más material orgánico se adhería en la superficie.

Por su parte, en el año 2009, fueron caracterizados los efectos de resistencia al ataque químico (manchado) y limpieza de dos baldosas con esmalte mate y una con esmalte brillante depositadas por el método de inmersión con películas de $\mathrm{TiO}_{2}$ y $\mathrm{ZrO}_{2}$ [23]; determinando que la eficiencia del recubrimiento de $\mathrm{TiO}_{2}$, que tiene una mayor capacidad de limpieza y autolimpieza después de haber sido expuesta a la luz UV. Los autores indican que la capacidad de limpieza de las superficies vidriadas brillantes no fue pronunciada, y que los recubrimientos de circonio podrían afectar negativamente la capacidad de autolimpieza de los esmaltes.

Hofer et al. [24] estudiaron la estabilidad térmica y la propiedad fotocatalítica activa del titanio al ser depositado sobre superficies cerámicas (sustratos esmaltados y sustratos de corindón no esmaltados) con área recubierta de $2,5 \times 4,0 \mathrm{~cm}$ utilizando un proceso por inmersión. encontrando que la reducción de la estabilidad térmica depende de la composición del sustrato y evidenciando una ligera modificación de la estructura electrónica del titanio, lo que indicó interacciones débiles entre la sílice y el titanio.

Similares resultados fueron reportados en el estudio de recubrimientos de baldosas cerámicas y vidrio de ventanas con películas delgadas de nano $\mathrm{TiO}_{2}$ utilizando técnicas de aplicación por dip y spray, demostrando un buen rendimiento de los recubrimientos para efectos de autolimpieza [25].

Adicional a ello, se ha usado para lograr efectos antibacterianos sobre sustratos vítreos de baldosas cerámicas empleando soluciones de $\mathrm{Ag} / \mathrm{SiO}_{2}$ [26] depositadas sobre la superficie esmaltada de las baldosas contra Escherichia coli y Staphylococcus aureus uti- 
lizando una técnica de recubrimiento por inmersión. Ellos encontraron un excelente rendimiento antibacteriano contra los dos tipos de bacterias, relacionado con la alta temperatura de sinterización de la capa depositada, ya que, después de este tratamiento los iones de plata se liberan constantemente, lo que genera cambios en las concentraciones de estos iones e inhiben el desarrollo de estas bacterias.

Soares et al. [27] utilizaron la técnica sol-gel para la producción de recubrimientos gruesos de partículas coloides de sílice y sílice/circonio sobre sustratos vitrocerámicos del sistema $\mathrm{Li}_{2} \mathrm{O}-\mathrm{Al}_{2} \mathrm{O}_{3}-\mathrm{SiO}_{2}$ con el fin de mejorar su resistencia al rayado y al desgaste. La deposición se realizó a una velocidad de extracción de $5 \mathrm{~cm} / \mathrm{min}$. Al comparar los resultados obtenidos con las propiedades del sustrato sin recubrir, se encontró una mejora en el aspecto final de la superficie para los recubrimientos funcionales; aunque, la resistencia al desgaste se mantuvo constante en comparación con las muestras sin recubrir.

Además, Zhang et al. [28] investigaron los efectos de la incorporación de nano partículas de titanio a soluciones de tetraetil ortosilicato (TEOS) depositadas sobre baldosas cerámicas vidriadas comerciales blancas $(25 \times 25 \times 5 \mathrm{~mm})$ mediante una técnica de recubrimiento por inmersión, en la cual se sumergieron los sustratos durante 3 minutos y luego se extrajeron a $1 \mathrm{~mm} / \mathrm{s}$. Esta investigación comprobó una mejor dispersión para las partículas de $\mathrm{P} 25-\mathrm{TiO}_{2} / \mathrm{TEOS}$, con mayor área superficial, mayor rugosidad de la superficie y un tamaño de partícula más pequeño en comparación con las partículas de $\mathrm{P} 25-\mathrm{TiO}_{2}$, lo que generó una mejor hidrofilicidad y una mayor actividad fotocatalítica bajo irradiación de luz visible.

López et al. [29] examinaron el efecto de diferentes tipos de sustratos sobre la morfología de la superficie y la fotocatálisis de película de $\mathrm{TiO}_{2}$ a gran escala. La impregnación se realizó mediante inmersión, específicamente, para el caso de los sustratos de vidrio de soda-cal esmerilado con ácido $(12 \times 21,5 \mathrm{~cm})$ se depositó una capa de barrera de $\mathrm{SiO}_{2}$ con una velocidad de extracción de $5 \mathrm{~cm} / \mathrm{min}$. Por su parte, los sustratos $(12 \times 21,5 \mathrm{~cm})$ de vidrio sódico-cal glaseado con ácido, vidrio de sosa-cal glaseado con ácido previamente recubierto con una capa de barrera de $\mathrm{SiO}_{2}$, baldosa cerámica esmaltada y una aleación de aluminio 6061, se impregnaron con una película de $\mathrm{TiO}_{2}$. Estos ensayos permitieron determinar que la fase y la microestructura presente en la película de $\mathrm{TiO}_{2}$ es exclusivamente anatasa; además de una variación sustancial del crecimiento de los cristales de $\mathrm{TiO}_{2}$, la morfología y el espesor de la película con respecto al sustrato usado, lo que a su vez afecta la actividad fotocatalítica.

Los estudios realizados por Hazmaliza et al. [30] reportan el rendimiento antibacteriano de la anatasa mezclada con esmalte y aplicada sobre baldosas cerámi- cas, utilizando dos tamaños diferentes de polvo de anatasa (micrométrico y nano). Para la realización de esta experimentación, se varió la concentración en peso del polvo de anatasa en micras, mientras que la anatasa de tamaño nano se estableció en un valor fijo. Los resultados obtenidos visualizan el cambio ligero del color de la baldosa producida, que se torna más amarillenta cuando aumenta la composición de anatasa; asimismo, evidencian que las propiedades antibacterianas aumentan al tener mayor composición de anatasa nanométrica en comparación con la micrométrica, debido a la mayor superficie de los agentes antibacterianos en la baldosa. Igualmente, entre mayor sea la composición de los polvos de anatasa, mayor será la viscosidad del esmalte.

Cabe anotar que mediante esta técnica también se ha investigado la aplicación de recubrimientos autolimpiantes de $\mathrm{TiO}_{2}$ sobre muestras de tejas de arcilla $(1 \times 1 \times 0,8 \mathrm{~cm})$ [31]. Los autores demostraron que un recubrimiento fotocatalítico de $\mathrm{TiO}_{2}$, en la fase nanocristalina anatasa, para película delgada (0,399 mg de suspensión / superficie de baldosa $\left.\mathrm{cm}^{2}\right)$, aplicada bajo condiciones industriales, tiene una mejor actividad fotocatalítica en la descomposición de azul de metileno, hidrofilicidad y actividad antimicrobiana que un recubrimiento fotocatalítico grueso $(0,885 \mathrm{mg}$ suspensión / $\mathrm{cm}^{2}$ ).

\subsection{Aspersión (Spray)}

Esta técnica de aplicación consiste en un proceso de aspersión o atomización vertical de una solución líquida estacionaria o en movimiento lineal, en donde un haz de fluido impulsado por aire comprimido se fragmenta en gotas dispersas que luego impactan sobre un sustrato. El spray tiene varias variables para su correcto funcionamiento, como son el caudal de la solución, la presión del aire comprimido, el tipo de boquilla y la distancia boquilla-sustrato, entre otras [32]. Dichas variables influyen en el tamaño, la forma, la velocidad y la fuerza de impacto de la gota; lo que también se encuentran ligadas a las propiedades del fluido (tensión superficial, densidad y viscosidad).

Después del impacto, se genera una transferencia de calor, entre el aire circundante y las gotas, que provoca la evaporación del solvente a través de la capa límite que rodea cada gota. Al finalizar la evaporación, las gotas se adhieren a la superficie vítrea creando un recubrimiento de capa delgada con espesor entre $0.1 \mathrm{y}$ $3 \mu \mathrm{m}[18]$.

Esta técnica de deposición no tiene limitación en el tamaño del sustrato pues el ángulo de abertura de la pistola de aplicación se regula. Requiere de poca cantidad de solución ya que su deposición se ejecuta solo en la superficie esmaltada reduciendo costos a su vez y minimizando el impacto ambiental puesto que se hace innecesario el uso de vacío para eliminar los gases emergentes del proceso [33-35]. 
Bondioli et al. (2009) [36] estudiaron la funcionalización de las superficies de las baldosas cerámicas industriales mediante la técnica sol-gel para mejorar la capacidad de limpieza del gres porcelánico fino sin esmaltar, preparando recubrimientos de $\mathrm{TiO}_{2}-\mathrm{SiO}_{2}$ con diferentes concentraciones de titanio que se depositaron mediante aerógrafo. En dicho trabajo reporta que las películas son trasparentes, no modifican el brillo del gres y muestran una buena adhesión; además de obtener un mayor efecto fotocatalítico a altas temperaturas de sinterización, lo que genera una superficie cerámica autolimpiante y autoesterilizante que, además, podría degradar varios contaminantes orgánicos en el ambiente circundante.

Savvova et al. [37] desarrollaron recubrimientos de $\mathrm{TiO}_{2}$ sobre esmalte cerámico, determinando la multifuncionalidad de este material al reportar su propiedad antibacteriana y antimicótica sustanciales de 70 al $90 \%$. Nuevamente, Bondioli et al. $[38,39]$ reportaron la funcionalización de las superficies de baldosas cerámicas, pero esta vez enfocado en sales solubles (procesos alternativos de coloración de materiales cerámicos sin esmaltar) para mejorar propiedades mecánicas (resistencia al rayado y al desgaste) y la conductividad.

Para ello, prepararon soluciones con diferentes concentraciones de circonio, titanio y plata; de las cuales se aplicaron con aerógrafo $300 \mathrm{~g} / \mathrm{cm}^{2}$ sobre bizcochos en crudo verde. Se visualizó que la adición de sales solubles en circonio aumentó la resistencia al rayado, al tiempo que mantiene la apariencia estética final de las baldosas cerámicas. Los resultados encontrados para titanio y plata fueron semejantes a los ya mencionados para circonio, con la diferencia de la generación de una capa multifuncional.

\subsection{Pantalla o serigrafía (Screen)}

La serigrafía o impresión en pantalla permite la realización de diseños bidimensionales, lo que le da utilidad en procesos industriales [40]. Los procesos serigráficos requieren de tejido que puede ser fibra sintética o malla de acero tensionado y pegado en un marco, el cual se cubre con una emulsión que es impermeable a la solución de recubrimiento en el área donde no debe aparecer ninguna impresión.

Es una técnica que prácticamente no ocasiona pérdidas de la solución de recubrimiento durante la impresión, pues el bastidor recorre la pantalla a una velocidad constante y al realizar la aplicación retrocede para repetir el procedimiento.

Debido a su versatilidad, este método ha sido uti- lizado desde hace 50 años en la industria cerámica para depositar esmaltes y decorar baldosas [18]. Para este tipo de sustratos, en procesos que generan capa delgada de $\mathrm{TiO}_{2}$ por la técnica sol-gel, produce espesores de 5 a $35 \mu \mathrm{m}$; teniendo en cuenta que tanto el espesor como la cantidad de material aplicado puede variar según el porcentaje de aplicación de la pantalla, que incluye el volumen entre los hilos de la pantalla y el espesor de la emulsión [40]. Ya que no se deposita todo el material en la pantalla, el espesor de la capa húmeda también depende de la fuerza y la velocidad de arrastre del bastidor, la distancia pantalla-sustrato, y la viscosidad de la solución depositada [40].

En el 2008, São Marcos et al. [41] estudiaron las capas de $\mathrm{TiO}_{2}$ depositadas a 25 y $50 \mathrm{~g} / \mathrm{m}^{2}$ mediante serigrafía (tamizado 55 y $136 \mu \mathrm{m}$ ) sobre baldosas monoporosas esmaltadas. Ellos determinaron que las capas otorgaban más del $90 \%$ de eficiencia después de 6 a 8 h de realización de la decoloración de Orange II bajo luz artificial visible sin desactivación de la capa; obteniendo niveles de decoloración aceptabas en periodos de tiempo razonables.

En el año siguiente, estos investigadores [42] caracterizaron y determinaron la degradación fotocatalítica del Orange II, para capas activas de $\mathrm{ZnO}$ depositadas a 25 y $50 \mathrm{~g} / \mathrm{m}^{2}$ mediante distintas pantallas tamizadas (55 y $136 \mu \mathrm{m}$ ) utilizando baldosas monoporosas con esmalte brillante $(20 \times 10 \mathrm{~cm}$ de Revigrés, PT); en este caso, los resultados revelaron un rendimiento de decoloración de Orange II considerable (más del $50 \%$ de eficiencia después de 6 a 8 h, con un grado de atenuación máximo del $72 \%$ ), bajo luz visible artificial o por exposición directa a luz del sol. Por ello, las capas de $\mathrm{TiO}_{2}$ y $\mathrm{ZnO}$ evitan la eliminación del fotocatalizador al final del proceso.

Para el mismo año, Rego et al. [43] utilizaron los dos materiales descritos para determinar la degradación fotocatalítica del colorante Orange II en soluciones acuosas, bajo exposición directa a la luz solar. Para ello, las capas de $\mathrm{TiO}_{2}$ y $\mathrm{ZnO}$ se depositaron con $50 \mathrm{~g} / \mathrm{m}^{2}$ sobre baldosas cerámicas de esmalte monoporoso brillante comerciales $(20 \times 20 \mathrm{~cm}$ de Revigrés, $\mathrm{PT}$ ), mediante un proceso serigráfico donde se usaron pantallas tamizadas $(136 \mu \mathrm{m})$.

Estas capas mostraron un rendimiento superior para el material $\mathrm{TiO}_{2}$; además de un grado máximo de atenuación del color del $90 \%$. Por su parte, en el año 2011 Seabra et al. [44] al evaluar el comportamiento fotocatalítico de capas de titanio depositadas por spray y serigrafía, encontró una mejor cobertura con niveles de rugosidad bajos para el método de spray. 
Tabla 3. Características y trabajos publicados según técnica de deposición

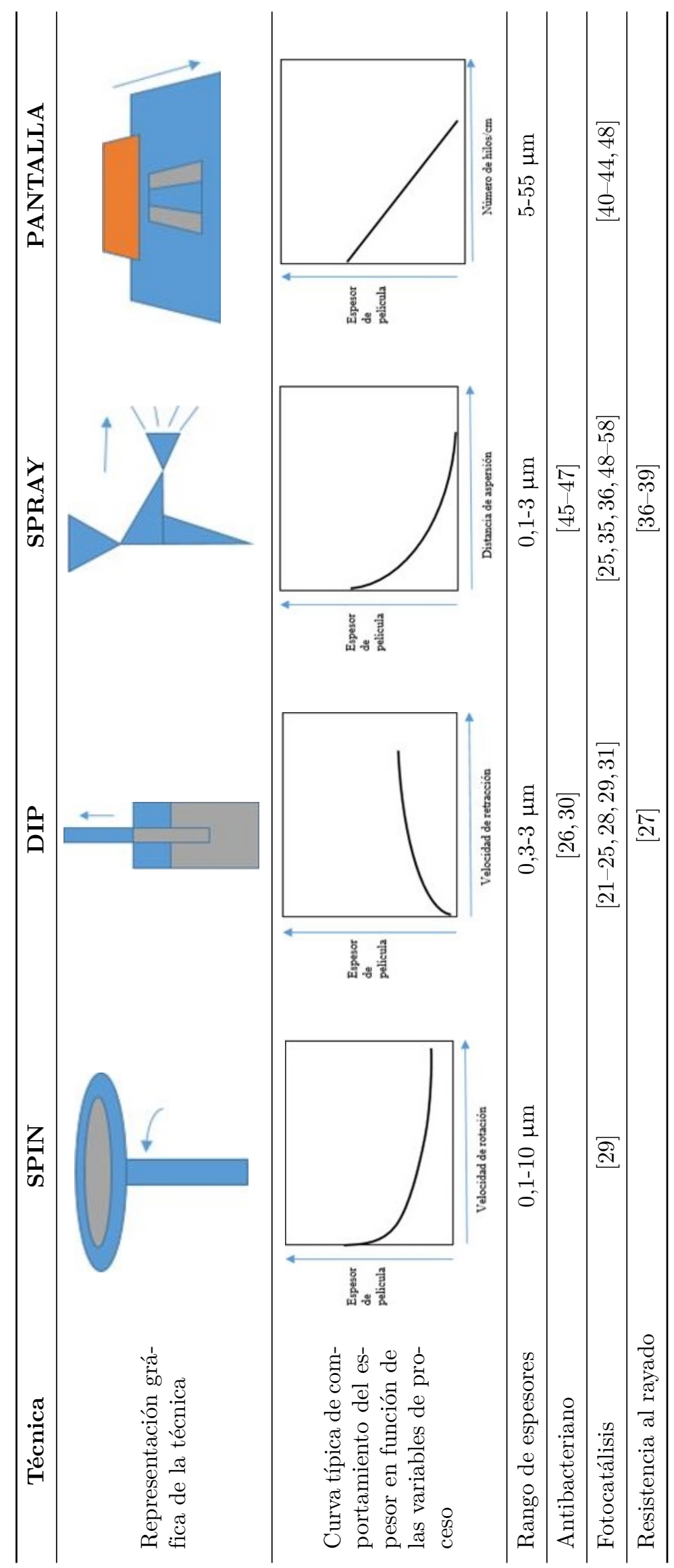




\section{Aplicaciones}

En la Figura 4 se presenta el gráfico de distribución de las investigaciones estudiadas según la aplicación deseada. Claramente se encuentra que las aplicaciones asociadas a la fotocatálisis y el efecto antibacteriano han sido el principal foco de atención para esta clase de desarrollos investigativos. Menos del $10 \%$ de estas investigaciones se han enfocado en incrementar la resistencia al rayado de las superficies. A continuación, se presentan una descripción breve de esta clase de aplicaciones.

\subsection{Fotocatálisis}

La absorción de fotones provenientes de la luz con suficiente energía (igual o superior a la banda energética del catalizador) promueve reacciones catalíticas en la superficie del material, generando excitones (pares $\mathrm{e}^{-}-$ $\mathrm{h}^{+}$). Dichos excitones pueden aprovecharse para llevar a cabo reacciones redox [59].

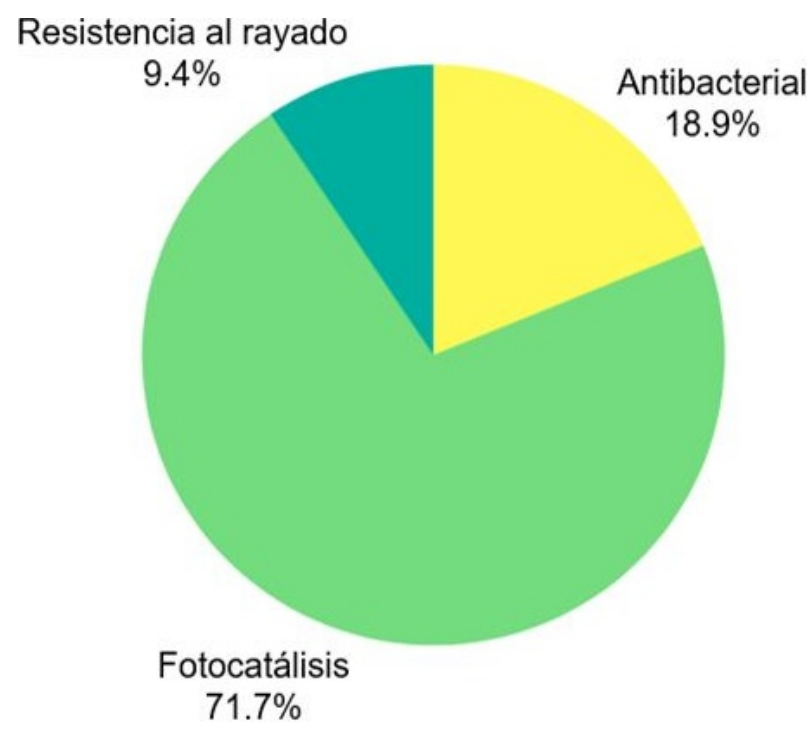

Figura 4. Distribución de investigaciones según aplicación.

El principal propósito de la obtención de recubrimientos con funciones catalíticas empleando titanio o variaciones de titanio en superficies cerámicas esmaltadas, es la autolimpieza tal y como es reportado en la revisión realizada por parte [18]. Esta característica es la combinación de dos fenómenos: la acción de catálisis sobre determinadas sustancias $\left(\mathrm{CO}_{2}, \mathrm{COVs}\right)$ y la superhidrofobicidad propia del titanio. Algunos autores también aseguran la posible eliminación de $\mathrm{NO}_{x}, \mathrm{CO}$, $\mathrm{SO}_{x}$ del aire $[60,61]$.

\subsection{Antibacteriano}

Uno de los efectos derivados de las interacciones entre radicales libres (iones y excitones) depositados en las superficies es la reducción significativa del crecimiento de microorganismos. El mecanismo de reducción radica en el bloqueo de la actividad aeróbica del microorganismo por ausencia de oxígeno disponible en el medio.

Esta aplicación ha resultado de alto interés para sitios públicos altamente expuestos a condiciones de crecimiento de microorganismos, como son centros de salud (clínicas, hospitales, salas de cirugía), escuelas y colegios tal y como lo han propuesto [62].

\subsection{Resistencia al rayado}

Detrás del incremento de esta propiedad subyacen aspectos relacionados con el incremento en otras propiedades como son la vida útil de las superficies y de los atributos que en ella se encuentran, a saber: brillo, color, textura, dureza, entre otros, los cuales son afectados por el desgaste consecuencia de los fenómenos de fricción a los cuales están sometidos los productos cerámicos.

La posibilidad de formar capas cerámicas nano y micrométricas, con estructura cristalina en vez de la estructura amorfa propia de los esmaltes vítreos, ha demostrado incrementos notorios en la respuesta de la superficie en lo que se refiere a la resistencia al rayado [63].

\section{Conclusiones}

Luego de la revisión efectuada se encontró que la técnica de sol-gel ha sido empleada para la fabricación de soluciones que se depositan en capas finas sobre los esmaltes de cerámica tradicional con el fin de aportar características funcionales al mismo. Las publicaciones encontradas corresponden a investigadores ubicados en Italia, Brasil, Portugal, principalmente. Las superficies con atributos funcionales de fotocatálisis (autolimpieza) han sido un eje importante que ha motivado las investigaciones en esta área. Siendo el amplio mercado y el potencial efecto positivo elementos de innovación para la industria.

Aun cuando es un tema cuyos orígenes investigativos datan del año 2000 y encuentra un fuerte impulso entre los años 2007 en adelante, las investigaciones en otras funcionalidades parecen estar susceptibles de ser estudiadas dado que es posible recubrir los esmaltes con soluciones alcóxidicas mediante la técnica de solgel.

\section{Referencias}

[1] O. J. Restrepo Baena, Baldosas cerámicas y gres porcelánico: un mundo en permanente evolución, C. E. F. de Minas, Ed. Universidad Nacional de Colombia, Sede Medellín, 2011. [Online]. Available: https://goo.gl/sSTprJ 
[2] L. Fröberg and L. Hupa, "Topographic characterization of glazed surfaces," $A p$ plied Surface Science, vol. 254, no. 6, pp. 1622-1629, 2008. [Online]. Available: https://doi.org/10.1016/j.apsusc.2007.07.173

[3] R. Casasola, J. M. Rincón, and M. Romero, "Glass-ceramic glazes for ceramic tiles: a review," Journal of Materials Science, vol. 47, no. 2, pp. 553-582, Jan 2012. [Online]. Available: https://doi.org/10.1007/s10853-011-5981-y

[4] A. Moreno Berto, "Ceramic tiles: Above and beyond traditional applications," Journal of the European Ceramic Society, vol. 27, no. 2, pp. 1607-1613, 2007. [Online]. Available: https: //doi.org/10.1016/j.jeurceramsoc.2006.04.146

[5] M. Raimondo, G. Guarini, C. Zanelli, F. Marani, L. Fossa, and M. Dondi, "Printing nano tio $_{2}$ on large-sized building materials: Technologies, surface modifications and functional behaviour," Ceramics International, vol. 38, no. 6, pp. 4685-4693, 2012. [Online]. Available: https://doi.org/10.1016/j.ceramint.2012.02.051

[6] J. González Hernández, J. Pérez Robles, F. Ruiz, and J. Martínez, "Vidrios $\mathrm{SiO}_{2}$ nanocompuestos preparados por sol-gel: revisión," Superficies y vacío, no. 11, pp. 1-16, 2000. [Online]. Available: https://goo.gl/cVrCwj

[7] C. J. Brinker and G. W. Scherer, SolGel Science: The Physics and Chemistry of Sol-Gel Processing. San Diego: Academic Press, 1990. [Online]. Available: https: //doi.org/10.1016/B978-0-08-057103-4.50007-6

[8] M. Guglielmi and G. Carturan, "Precursors for sol-gel preparations," Journal of Non-Crystalline Solids, vol. 100, no. 1, pp. 16-30, 1988. [Online]. Available: https://doi.org/10.1016/0022-3093(88)90004-X

[9] J. Livage and D. Ganguli, "Sol-gel electrochromic coatings and devices: A review," Solar Energy Materials and Solar Cells, vol. 68, no. 3, pp. 365-381, 2001. [Online]. Available: https://doi.org/10.1016/S0927-0248(00)00369-X

[10] J. Livage, "Sol-gel processes," Current Opinion in Solid State and Materials Science, vol. 2, no. 2, pp. 132-138, 1997. [Online]. Available: https://doi.org/10.1016/S1359-0286(97)80057-5

[11] L. Znaidi, "Sol-gel-deposited zno thin films: A review," Materials Science and Engineering: B, vol. 174, no. 1, pp. 18-30, 2010. [Online]. Available: https://doi.org/10.1016/j.mseb.2010.07.001
[12] D. Chen, "Anti-reflection (ar) coatings made by sol-gel processes: A review," Solar Energy Materials and Solar Cells, vol. 68, no. 3, pp. 313-336, 2001. [Online]. Available: https://doi.org/10.1016/S0927-0248(00)00365-2

[13] S. Attia, J. Wang, G. Wu, J. Shen, and J. Ma, "Review on sol-gel derived coatings: Process, techniques and optical applications," Journal of Materials Science \&6 Technology, vol. 18, no. 3, pp. 211-218, 2002. [Online]. Available: https: //doi.org/10.3321/j.issn:1005-0302.2002.03.005

[14] D. Meyerhofer, "Characteristics of resist films produced by spinning," Journal of Applied Physics, vol. 49, no. 7, pp. 3993-3997, 1978. [Online]. Available: https://doi.org/10.1063/1.325357

[15] J. H. Lai, "An investigation of spin coating of electron resists," Polymer Engineering $\&$ Science, vol. 19, no. 15, pp. 1117-1121, 1979. [Online]. Available: https://doi.org/10.1002/pen.760191509

[16] J. Martín-Márquez, J. M. Rincón, and M. Romero, "Effect of firing temperature on sintering of porcelain stoneware tiles," Ceramics International, vol. 34, no. 8, pp. 1867-1873, 2008. [Online]. Available: https://doi.org/10.1016/j.ceramint.2007.06.006

[17] D. B. Hall, P. Underhill, and J. M. Torkelson, "Spin coating of thin and ultrathin polymer films," Polymer Engineering \& Science, vol. 38, no. 12, pp. 2039-2045, 1998. [Online]. Available: https://doi.org/10.1002/pen.10373

[18] A. L. da Silva, M. Dondi, M. Raimondo, and D. Hotza, "Photocatalytic ceramic tiles: Challenges and technological solutions," Journal of the European Ceramic Society, vol. 38, no. 4, pp. 1002-1017, 2018. [Online]. Available: https: //doi.org/10.1016/j.jeurceramsoc.2017.11.039

[19] C. A. Otálora Bastidas, "Desarrollo de materiales usados en la fabricación de celdas solares orgánicas," Master's thesis, Universidad Nacional de Colombia, Bogotá, Colombia, 2013. [Online]. Available: https://goo.gl/4kKihE

[20] C. Terrier, J. Chatelon, R. Berjoan, and J. Roger, "Sb-doped $\mathrm{SnO}_{2}$ transparent conducting oxide from the sol-gel dip-coating technique," Thin Solid Films, vol. 263, no. 1, pp. 37-41, 1995. [Online]. Available: https://doi.org/10.1016/0040-6090(95)06543-1

[21] S. Kato, S. Kato, H. Taoda, and S. Katoh, "Thin film coating of photocatalytics on ultra light ceramic tile by use of supercritical fluid," High Pressure Research, vol. 20, no. 
1-6, pp. 415-419, 2001. [Online]. Available: https://doi.org/10.1080/08957950108206189

[22] R. Kuisma, L. Fröberg, H.-R. Kymäläinen, E. Pesonen-Leinonen, M. Piispanen, P. Melamies, M. Hautala, A.-M. Sjöberg, and L. Hupa, "Microstructure and cleanability of uncoated and fluoropolymer, zirconia and titania coated ceramic glazed surfaces," Journal of the European Ceramic Society, vol. 27, no. 1, pp. 101-108, 2007. [Online]. Available: https: //doi.org/10.1016/j.jeurceramsoc.2006.02.035

[23] M. Piispanen, J. Määttä, S. Areva, A.-M. Sjöberg, M. Hupa, and L. Hupa, "Chemical resistance and cleaning properties of coated glazed surfaces," Journal of the European Ceramic Society, vol. 29, no. 10, pp. 1855-1860, 2009. [Online]. Available: https: //doi.org/10.1016/j.jeurceramsoc.2008.11.007

[24] M. Hofer and D. Penner, "Thermally stable and photocatalytically active titania for ceramic surfaces," Journal of the European Ceramic Society, vol. 31, no. 15, pp. 2887-2896, 2011. [Online]. Available: https: //doi.org/10.1016/j.jeurceramsoc.2011.07.016

[25] K. Murugan, R. Subasri, T. Rao, A. S. Gandhi, and B. Murty, "Synthesis, characterization and demonstration of self-cleaning tio2 coatings on glass and glazed ceramic tiles," Progress in Organic Coatings, vol. 76, no. 12 , pp. 1756-1760, 2013. [Online]. Available: https://doi.org/10.1016/j.porgcoat.2013.05.012

[26] N. Baheiraei, F. Moztarzadeh, and M. Hedayati, "Preparation and antibacterial activity of $\mathrm{Ag} / \mathrm{SiO}_{2}$ thin film on glazed ceramic tiles by sol-gel method," Ceramics International, vol. 38, no. 4, pp. 2921-2925, 2012. [Online]. Available: https://doi.org/10.1016/j.ceramint.2011.11.068

[27] V. Soares, P. Soares, O. Peitl, E. Zanotto, A. Durán, and Y. Castro, "Resistencia al desgaste de recubrimientos sol-gel de $\mathrm{SiO}_{2}$ y $\mathrm{SiO}_{2}-\mathrm{ZrO}_{2}$ sobre materiales vitrocerámicos obtenidos por sinterización," Boletín la Sociedad Española De Cerámica y Vidrio, vol. 52, no. 5, pp. 225-230, 2013. [Online]. Available: https://doi.org/10.3989/cyv.272013

[28] P. Zhang, J. Tian, R. Xu, and G. Ma, "Hydrophilicity, photocatalytic activity and stability of tetraethyl orthosilicate modified tio2 film on glazed ceramic surface," Applied Surface Science, vol. 266, pp. 141-147, 2013. [Online]. Available: https://doi.org/10.1016/j.apsusc.2012.11.117
[29] L. Lopez, W. A. Daoud, D. Dutta, B. C. Panther, and T. W. Turney, "Effect of substrate on surface morphology and photocatalysis of large-scale $\mathrm{TiO}_{2}$ films," Applied Surface Science, vol. 265, pp. 162-168, 2013. [Online]. Available: https://doi.org/10.1016/j.apsusc.2012.10.156

[30] M. Hasmaliza, H. Foo, and K. Mohd, "Anatase as antibacterial material in ceramic tiles," Procedia Chemistry, vol. 19, pp. 828-834, 2016. [Online]. Available: https://doi.org/10.1016/j.proche.2016.03.109

[31] M. Hadnadjev, J. Ranogajec, S. Petrovic, S. Markov, V. Ducman, and R. MarinkovicNeducin, "Design of self-cleaning tio 2 coating on clay roofing tiles," Philosophical Magazine, vol. 90, no. 22 , pp. $2989-3002,2010$. [Online]. Available: https://doi.org/10.1080/14786431003767017

[32] B. Yu, D. Vak, J. Jo, S. Na, S. Kim, M. Kim, and D. Kim, "Factors to be considered in bulk heterojunction polymer solar cells fabricated by the spray process," IEEE Journal of Selected Topics in Quantum Electronics, vol. 16, no. 6, pp. 1838-1846, Nov 2010. [Online]. Available: https://doi.org/10.1109/JSTQE.2010.2042282

[33] F. Aziz and A. Ismail, "Spray coating methods for polymer solar cells fabrication: A review," Materials Science in Semiconductor Processing, vol. 39, pp. 416-425, 2015. [Online]. Available: https://doi.org/10.1016/j.mssp.2015.05.019

[34] S. Colella, M. Mazzeo, G. Melcarne, S. Carallo, G. Ciccarella, and G. Gigli, "Spray coating fabrication of organic solar cells bypassing the limit of orthogonal solvents," Applied Physics Letters, vol. 102, no. 20, p. 203307, 2013. [Online]. Available: https://doi.org/10.1063/1.4807464

[35] C. Sciancalepore and F. Bondioli, "Durability of $\mathrm{SiO}_{2}-\mathrm{TiO}_{2}$ Photocatalytic Coatings on Ceramic Tiles," International Journal of Applied Ceramic Technology, vol. 12, no. 3, pp. 679-684, 2015. [Online]. Available: https://doi.org/10.1111/ijac.12240

[36] F. Bondioli, R. Taurino, and A. Ferrari, "Functionalization of ceramic tile surface by sol-gel technique," Journal of Colloid and Interface Science, vol. 334, no. 2, pp. 195-201, 2009. [Online]. Available: https://doi.org/10.1016/j.jcis.2009.02.054

[37] O. V. Savvova and L. L. Bragina, "Use of titanium dioxide for the development of antibacterial glass enamel coatings," Glass and Ceramics, vol. 67, no. 5 , pp. 184-186, Nov 2010. [Online]. Available: https://doi.org/10.1007/s10717-010-9258-8 
[38] F. Bondioli, T. Manfredini, M. Giorgi, and G. Vignali, "Functionalization of ceramic tile surface by soluble salts addition: Part i," Journal of the European Ceramic Society, vol. 30, no. 1, pp. 11-16, 2010. [Online]. Available: https: //doi.org/10.1016/j.jeurceramsoc.2009.08.012

[39] F. Bondioli, M. Dinelli, R. Giovanardi, and M. Giorgi, "Functionalization of ceramic tile surface by soluble salts addition: Part ii. titanium and silver addition," Journal of the European Ceramic Society, vol. 30, no. 9, pp. 1873-1878, 2010. [Online]. Available: https: //doi.org/10.1016/j.jeurceramsoc.2010.03.008

[40] F. C. Krebs, "Fabrication and processing of polymer solar cells: A review of printing and coating techniques," Solar Energy Materials and Solar Cells, vol. 93, no. 4, pp. 394-412, 2009. [Online]. Available: https://doi.org/10.1016/j.solmat.2008.10.004

[41] P. S. Marcos, J. Marto, T. Trindade, and J. Labrincha, "Screen-printing osol-g $\mathrm{g}_{2}$ photocatalytic layers on glazed ceramic tiles," Journal of Photochemistry and Photobiology A: Chemistry, vol. 197, no. 2 , pp. 125-131, 2008. [Online]. Available: https: //doi.org/10.1016/j.jphotochem.2007.12.017

[42] J. Marto, P. S. Marcos, T. Trindade, and J. Labrincha, "Photocatalytic decolouration of orange ii by zno active layers screen-printed on ceramic tiles," Journal of Hazardous Materials, vol. 163, no. 1, pp. 36-42, 2009. [Online]. Available: https://doi.org/10.1016/j.jhazmat.2008.06.056

[43] E. Rego, J. Marto, P. S. Marcos, and J. Labrincha, "Decolouration of orange ii solutions by tio2 and zno active layers screenprinted on ceramic tiles under sunlight irradiation," Applied Catalysis A: General, vol. 355, no. 1, pp. 109-114, 2009. [Online]. Available: https://doi.org/10.1016/j.apcata.2008.12.005

[44] M. Seabra, R. Pires, and J. Labrincha, "Ceramic tiles for photodegradation of orange ii solutions," Chemical Engineering Journal, vol. 171, no. 2, pp. 692-702, 2011. [Online]. Available: https://doi.org/10.1016/j.cej.2011.04.028

[45] A. Ghafari-Nazari, F. Moztarzadeh, S. M. Rabiee, T. Rajabloo, M. Mozafari, and L. Tayebi, "Antibacterial activity of silver photodeposited nepheline thin film coatings," Ceramics International, vol. 38, no. 7, pp. 5445-5451, 2012. [Online]. Available: https://doi.org/10.1016/j.ceramint.2012.03.055

[46] M. Machida, K. Norimoto, and T. Kimura, "Antibacterial activity of photocatalytic titanium dioxide thin films with photodeposited silver on the surface of sanitary ware," Journal of the American Ceramic Society, vol. 88, no. 1, pp. 95-100, 2005. [Online]. Available: https: //doi.org/10.1111/j.1551-2916.2004.00006.x

[47] J. Szczawiński, H. Tomaszewski, A. JackowskaTracz, and M. Szczawińska, "Survival of Staphylococcus aureus exposed to UV radiation on the surface of ceramic tiles coated with $\mathrm{TiO}_{2}, "$ Polish Journal of Veterinary Sciences, vol. 14, no. 1, pp. 41-46, 2011. [Online]. Available: https://doi.org/10.2478/v10181-011-0006-y

[48] C. L. Bianchi, B. Sacchi, S. Capelli, C. Pirola, G. Cerrato, S. Morandi, and V. Capucci, "Micro-sized $\mathrm{tio}_{2}$ as photoactive catalyst coated on industrial porcelain grès tiles to photodegrade drugs in water," Environmental Science and Pollution Research, vol. 25, no. 21, pp. 20348-20 353, Jul 2018. [Online]. Available: https://doi.org/10.1007/s11356-017-9066-6

[49] A. M. Buckley and M. Greenblatt, "The sol-gel preparation of silica gels," Journal of Chemical Education, vol. 71, no. 7, p. 599, 1994. [Online]. Available: https://doi.org/10.1021/ed071p599

[50] A. L. da Silva, M. Dondi, and D. Hotza, "Self-cleaning ceramic tiles coated with $\mathrm{Nb}_{2} \mathrm{O} 5$ doped- $\mathrm{TiO}_{2}$ nanoparticles," Ceramics International, vol. 43, no. 15 , pp. $11986-$ 11991, 2017. [Online]. Available: https: //doi.org/10.1016/j.ceramint.2017.06.049

[51] A. L. da Silva, D. N. Muche, S. Dey, D. Hotza, and R. H. Castro, "Photocatalytic $\mathrm{nb}_{2} \mathrm{O}_{5}$-doped $\mathrm{tio}_{2}$ nanoparticles for glazed ceramic tiles," Ceramics International, vol. 42, no. 4 , pp. 5113-5122, 2016. [Online]. Available: https://doi.org/10.1016/j.ceramint.2015.12.029

[52] S. Niederhãusern, M. Bondi, and F. Bondioli, "Self-cleaning and antibacteric ceramic tile surface," International Journal of Applied Ceramic Technology, vol. 10, no. 6, pp. 949-956, 2013. [Online]. Available: https: //doi.org/10.1111/j.1744-7402.2012.02801.x

[53] V. Ducman, V. Petrovič, and S. D. Škapin, "Photo-catalytic efficiency of laboratory made and commercially available ceramic building products," Ceramics International, vol. 39, no. 3, pp. 2981-2987, 2013. [Online]. Available: https://doi.org/10.1016/j.ceramint.2012.09.075

[54] S. Ke, X. Cheng, Q. Wang, Y. Wang, and Z. Pan, "Preparation of a photocatalytic $\mathrm{TiO}_{2} / \mathrm{ZnTiO}_{3}$ coating on glazed ceramic tiles," Ceramics International, vol. 40, 
no. 6 , pp. $8891-8895,2014$. [Online]. Available: https://doi.org/10.1016/j.ceramint.2014.01.027

[55] V. Petrovič, V. Ducman, and S. D. Škapin, "Determination of the photocatalytic efficiency of $\mathrm{TiO}_{2}$ coatings on ceramic tiles by monitoring the photodegradation of organic dyes," Ceramics International, vol. 38, no. 2, pp. 1611-1616, 2012. [Online]. Available: https://doi.org/10.1016/j.ceramint.2011.09.050

[56] P. Sooksaen, N. Saowaros, K. Ngamkaruhasereethorn, and A. Pringkasemchai, "Photocatalytic degradation study of titania sol-gel coated on commercial unglazed ceramic tiles," Key Engineering Materials, vol. 751, pp. 819-824, 2017. [Online]. Available: https://doi.org/10.4028/www. scientific.net/KEM.751.819

[57] V. B. Tezza, M. Scarpato, L. F. S. Oliveira, and A. M. Bernardin, "Effect of firing temperature on the photocatalytic activity of anatase ceramic glazes," Powder Technology, vol. 276, pp. 60-65, 2015. [Online]. Available: https://doi.org/10.1016/j.powtec.2015.01.076

[58] R. Taurino, L. Barbieri, and F. Bondioli, "Surface properties of new green building material after $\mathrm{tio}_{2} \mathrm{SiO}_{2}$ coatings deposition," Ceramics International, vol. 42, no. 4 , pp. $4866-4874,2016$. [Online]. Available: https://doi.org/10.1016/j.ceramint.2015.12.002

[59] M. Nevárez-Martínez, P. Espinoza-Montero, F. Quiroz-Chávez, and B. Ohtani, "Fotocatálisis: inicio, actualidad y perspectivas a través del tio 2 ," Avances en Química, vol. 12, no. 2-3, pp. 45-59, 2018. [Online]. Available: https://goo.gl/FtiWUw

[60] L. Liao, S. Heylen, S. P. Sree, B. Vallaey, M. Keulemans, S. Lenaerts, M. B. Roeffaers, and J. A. Martens, "Photocatalysis assisted simultaneous carbon oxidation and no $x$ reduction," Applied Catalysis B: Environmental, vol. 202, pp. 381-387, 2017. [Online]. Available: https://doi.org/10.1016/j.apcatb.2016.09.042

[61] Y. Boyjoo, H. Sun, J. Liu, V. K. Pareek, and S. Wang, "A review on photocatalysis for air treatment: From catalyst development to reactor design," Chemical Engineering Journal, vol. 310, pp. 537-559, 2017. [Online]. Available: https://doi.org/10.1016/j.cej.2016.06.090

[62] S. Q. Sun, B. Sun, W. Zhang, and D. Wang, "Preparation and antibacterial activity of ag-tio 2 composite film by liquid phase deposition (lpd) method," Bulletin of Materials Science, vol. 31, no. 1, pp. 61-66, Feb 2008. [Online]. Available: https://doi.org/10.1007/s12034-008-0011-7

[63] F. Tana, M. Messori, D. Contini, A. Cigada, T. Valente, F. Variola, L. D. Nardo, and F. Bondioli, "Synthesis and characterization of scratch-resistant hybrid coatings based on non-hydrolytic sol-gel $\mathrm{ZrO}_{2}$ nanoparticles," Progress in Organic Coatings, vol. 103, pp. 60-68, 2017. [Online]. Available: https://doi.org/10.1016/j.porgcoat.2016.11.022 